قبائل البوار في المغرب القديم على ضوء المصادر والنقوش اللاتينية

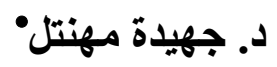

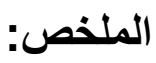

يعتبر البوار(Bavares) من القبائل المغاربية الكبيرة التي عرفت في الفترة القديمة،

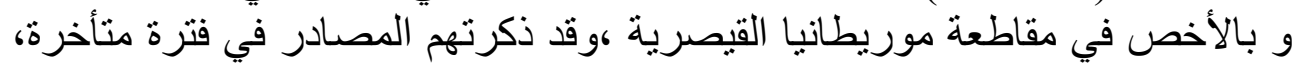

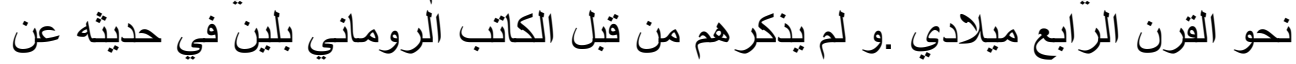

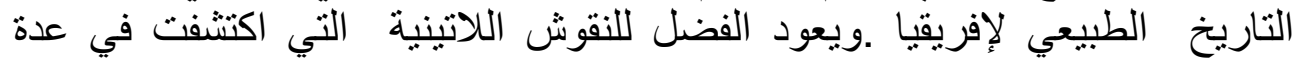

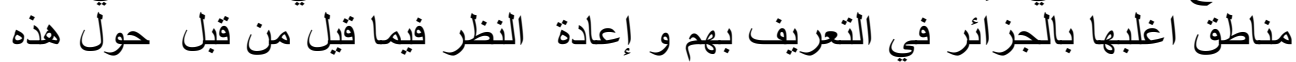

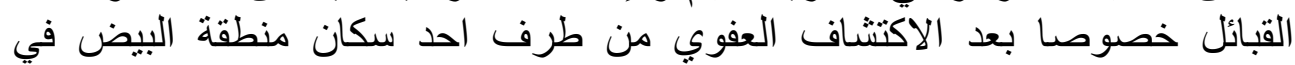

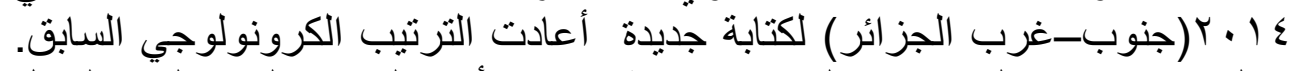

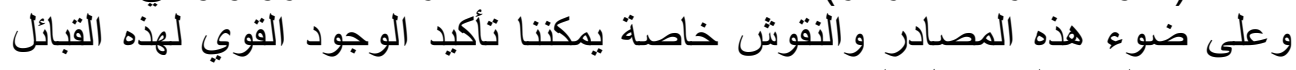
وتصديها الكبير للاحتلال الروماني.

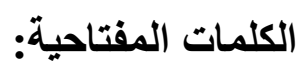
قبائل، بوار، مغاربية، قديمة، موريطانيا القيصرية، فترة متاخرة، نصوص ادبية، نقوش، البيض، مقاومة، رومان.

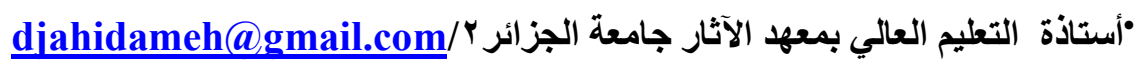


لقد جاء الاحتلال الروماني لبلاد المغرب القديم بصفة تدريجية، استعملت فيها روما لئها

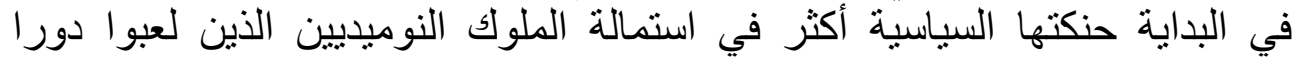

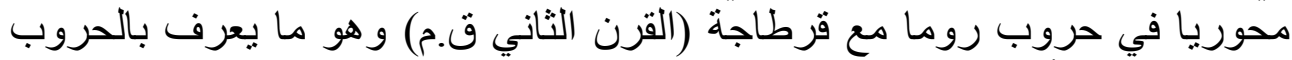

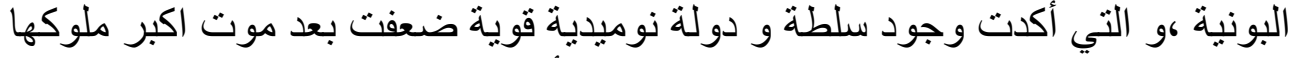

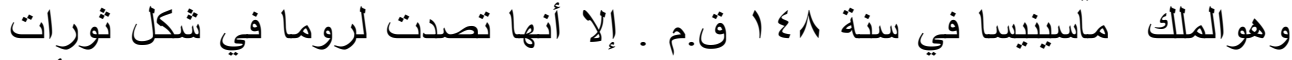

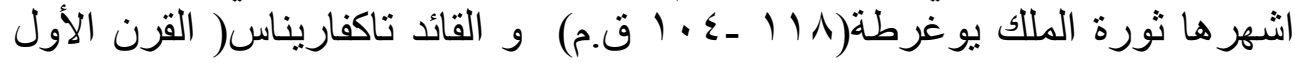
ميلادي) و غير هم. إلى جانب ثورة ثرات قامت بها مختلف القبائل المغاربية، كقبائل

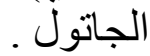

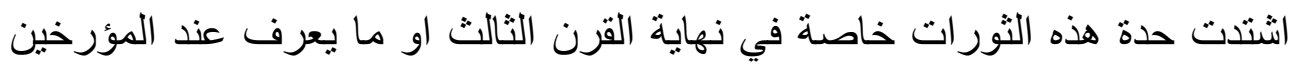

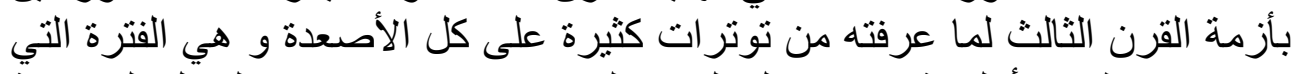

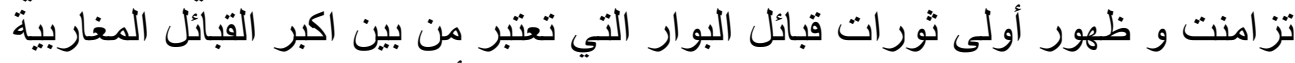

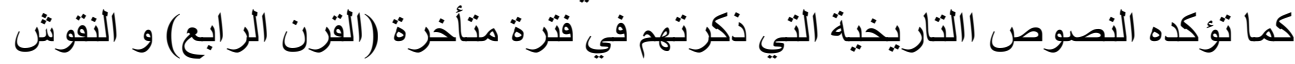

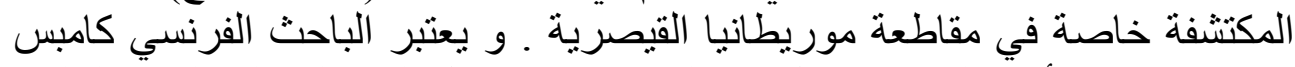
أهم الباحثين حول هذا الموضوع. وتثشل المداخلة على النقاط التالية النية (Camps .G)

ا ـقبائل البوار في المصادر التاريخية

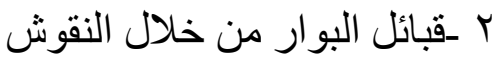

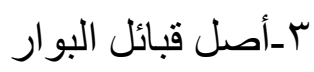
ع - ثورات البوار (حوصلة)

1 ـ قبائل البوار في المصادر الأدبية:

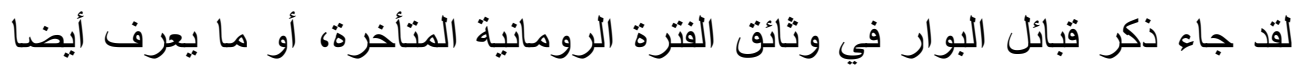

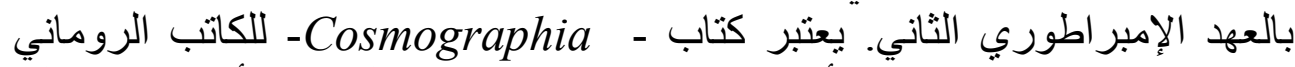
اونوريوس- L.Honorius- أهم وثيقة اثثوجر افية في هذه الفترة ، لأنه يحتوي على الألى

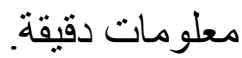

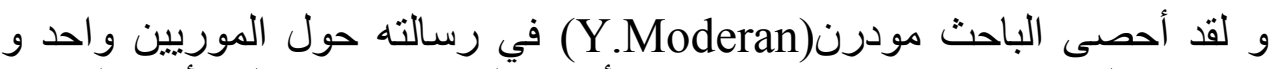

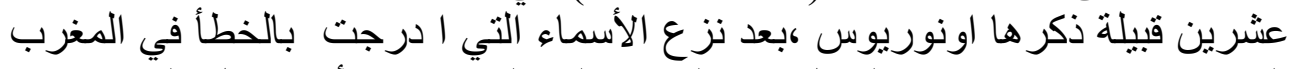

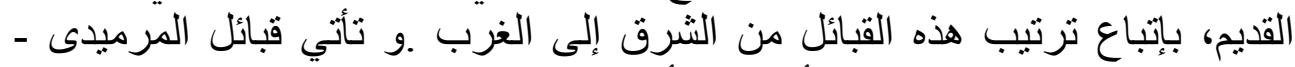
عidarmaridae عشر'

1. Moderan.Y,Les Maures et l'Afrique romaine(IV-VII siècle),Ecole française de Rome,2003,p154 


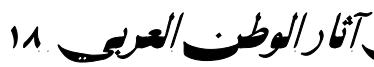

وتحدد فقرة من كتاب اونوريويس تواجدهم مع قبائل البقوات على طلى طرفي نهر الملوية

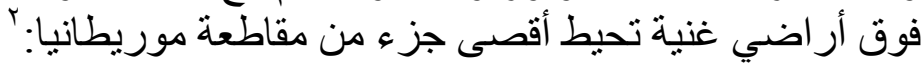

Fluvius Malva nascitur sub insulas Fortunatas circuiens extremam partem Mauretaniae, intrcludens inter Barbares et Bacuates Y(Riese,Geographi latini,1878,)

كما ذكرت هذه القبائل في كتاب الأجيال - liber Generationis - الذي يحصي الإني

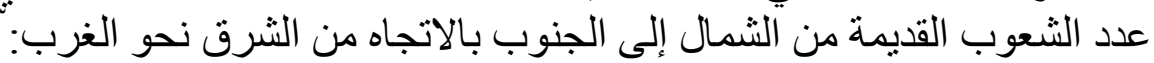

gentes autem quae suas habent sunt:Mauri Bacuates et Mssena, Gaetuli,Afri qui et Barbares r(liber Generationis, 1892)

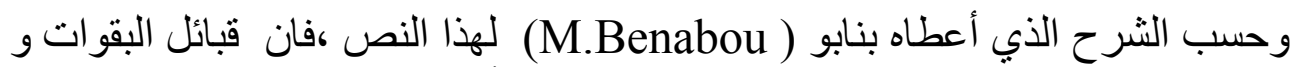

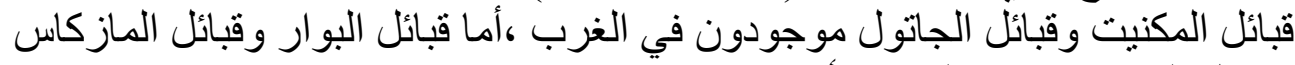

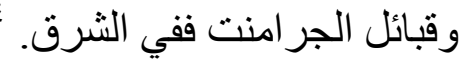

وتحددهم قائمة فيرون(liste de Verone) بين قبائل المازكاس وقبائل البقوات في الجزء الغربي لموريطانيا القيصرية: فيرون

item gentes quae in Mauretania sunt Mauri gentiani,Mauri Mazices Mauri barbares Mauri Bacuates ${ }^{0}$ (Riese,geographi latini, 1878,)

اما اميان مرسولان (Ammien Marcellin) فيكتب اسمهم بحرف الدال Daveres و يذكر هم بجوار قبائل المازكاس لَّاس

ويقى اونوريوس من بين أهم المصادر التي أثنارت إلى قبائل البوار حسب الكثير من الته

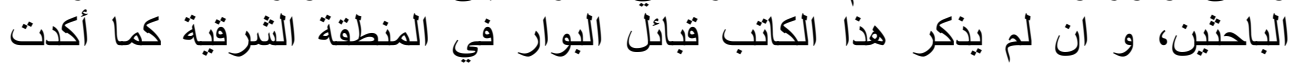

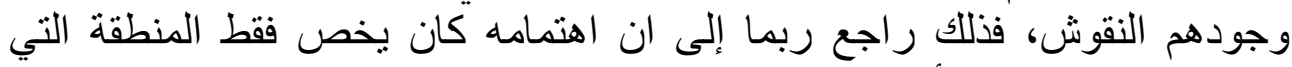

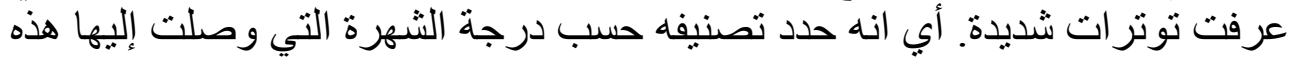

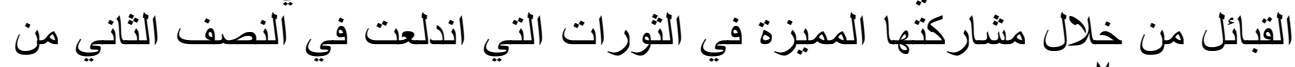

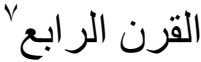

\footnotetext{
2 -Riese.A,in Geographi Latini minores,Berlin, 1878 ,p53

3 - liber Generationis, in chronica minora,edit,Mommsen, Berlin,1892,p165-167

${ }^{4}$ Benabou.M, La résistance africaine à la romanisation, Paris, 1976, p196

${ }^{5}$-Riese.A,op cit p129

${ }^{6}$ Marcellin.A, Histoire, XXIX, 5, 33, edit, M.A.Marie, Paris, 1984

${ }^{7}$ Moderan.Y,op. cit,p 119
} 


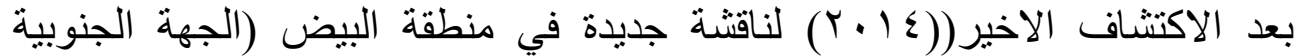

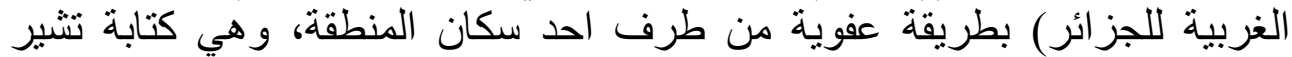

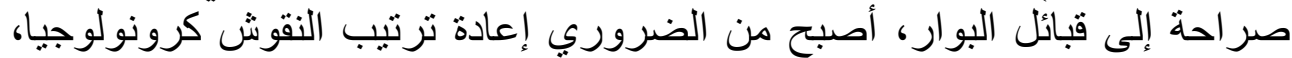

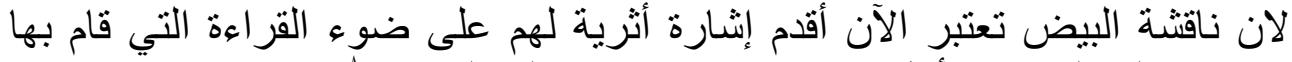
دريسي سليم الذي كان أول من استطاع الاطلاع على الأنى الناقتة .

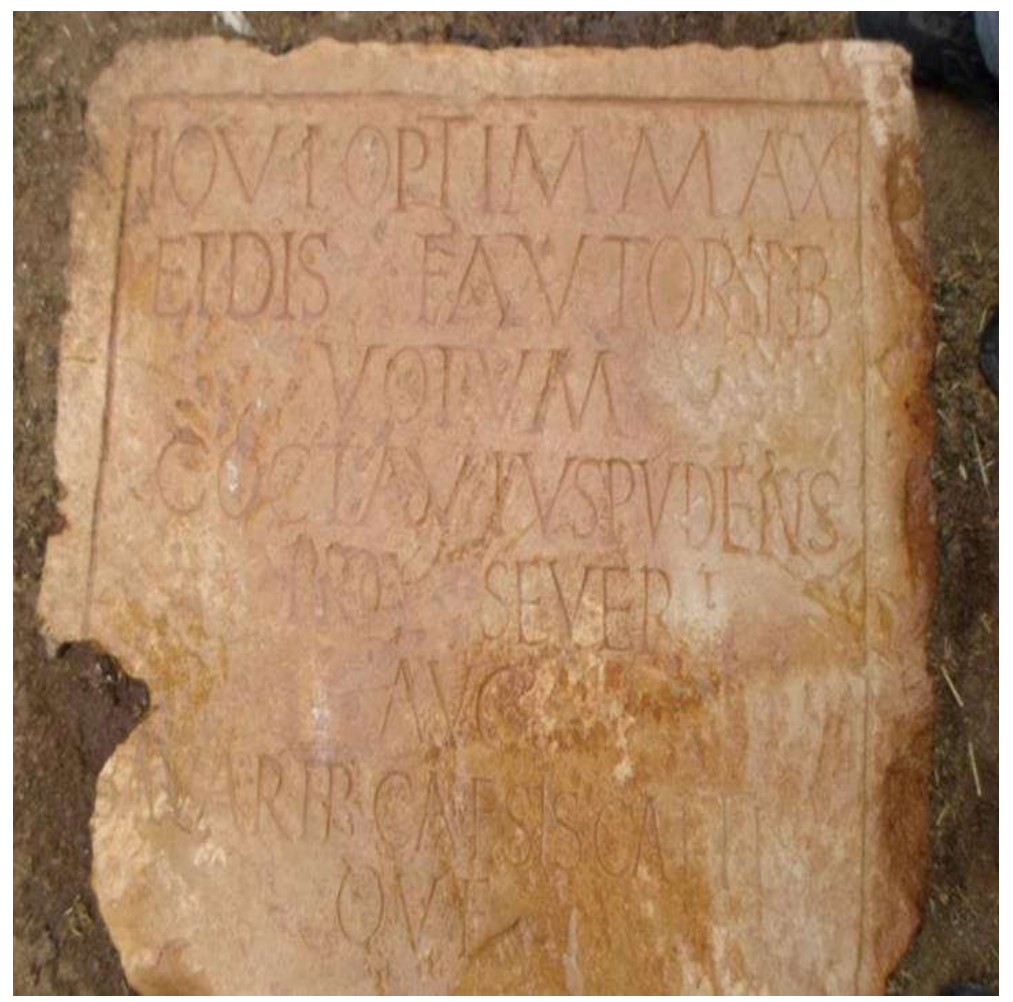

IOVI OPTIM (O) MAX (IMO)

ET DIS FAUTORIB(US)

VOTUM

C(AIUS) OCTAVIUS PUDENS

PROC (URATORI) SEVERI

AUG (USTI)

BAVARIB (US) CAESIS CAPTIS

QUE

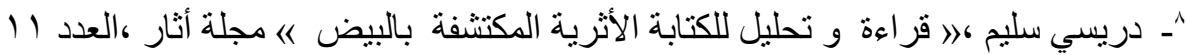

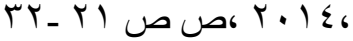


فان الناقشة عبارة عن إهداء إلى الإله يوبيتار الأعظم و الآلهة الحامية قام به حاكم

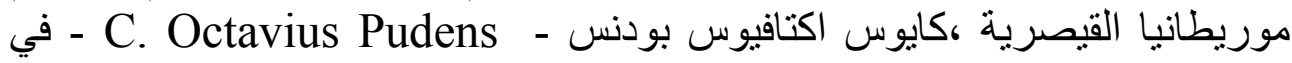

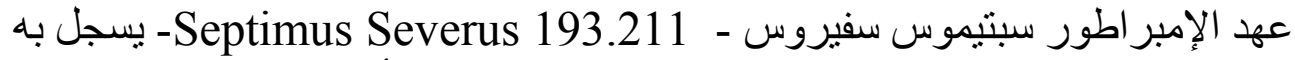
انتصاره على البوار الذين قتلوا و اسرو الكما تبينه العبارة الأخيرة من نص الكتابة.

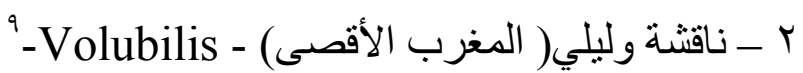

$\mathrm{I}($ ovi) $[\mathrm{O}$ (ptimo)M(aximo)]/ceterisq(ue)diis( !)d[eabus(que)immor talibus pro salute et incolumit(ate)]/et victoria imp(eratoris)C(aesaris) [M(arci) Aureli

SeveriAlexandri( ?)PiiFelicis/[A]ug(usti)Q(uintus)Herreni[us3]/v (ir)e(gregius)pro(urator)eius colloquiium $] /[\mathrm{cu}] \mathrm{m}[$ Au]relio(?)[3princ(ipe) gentis Bavarum et Baqua]/tium pa]cis firmand[ae gratia habuit aram(que) posuit et dedicavit $] /[$ idib(us)sep]tembribus i[mperatore)Alexandro Aug(usto)IIAufidio Marcello II co(n)s(ulibus ?)]

تتحدث الكتابة عن معاهدة سلم بين حاكم عسكري لا يظهر اسمه و بين قائد قبيلة

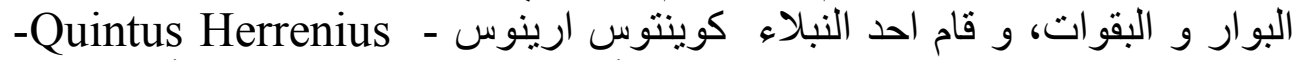

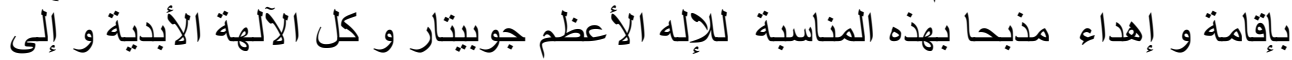

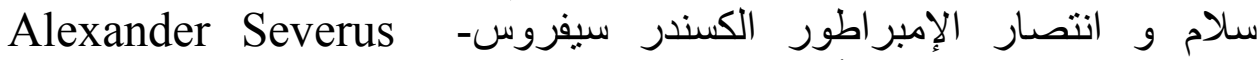
222.235- التقي و السعيد أغسطس الأورس يرى بنابو في وجود مساعد الحاكم العسكري ( pro légat) قلق روما تجاه هذه التوترات كما أننا نفهم من الكتابة ان قبائل البوار و قبائل البقوات هم قبيلة واحدة لان كلمة ـ جاءت في حالة المفرد. -gens 3 ناقشة لامبيز (شرق الجز ائر)- 'Lambeisis

I(ovi)O(ptimo)M(aximo)/ceterisq(ue)diis( !)deabusq(ue)immortal $\mathrm{ib}$ (us /C(aius)MacrinusDcianus $\quad \mathrm{v}$ (ir)c(arissimus) legat(us)/Augg(ustorum) pr(o)pr(aetore)prov(inciarum)Numidae et No/rici Bavaribus qui adunatisIIII/regibus in prov(inciam)Numidiam in/ruperant primum in

\footnotetext{
${ }^{9}$ IAM ,edit Gascou et Y.kich,Paris, 1982 , n-02-02,00356

${ }^{10}$ Benabou.M, op.cit, p197

${ }^{11} \mathrm{CIL}$, tome,VIII ,Berolini, 1863 n 02615
} 


\section{1} (4)

regione/Millevitana iterato in confi/nio Mauretaniae et Numidi/ae tertio Quinquegentaneis/gentilibus Mauretaniae Cae/sariensis item gentilibus Fra/xinensibus qui provinciam/Numidiam vastabant cap/to famosissimo duce eorum/caesisfugatisque

تعتبر أكثر أهية من حيث المعلومات التي توفر ها لنا،تتحدث عن إهداء قام بـه حاكم

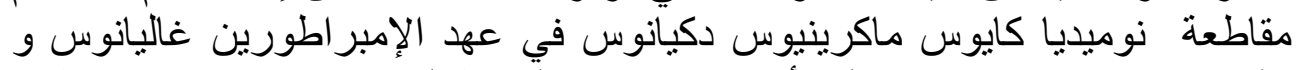

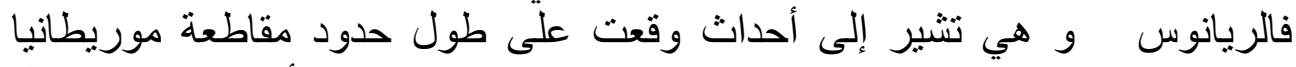

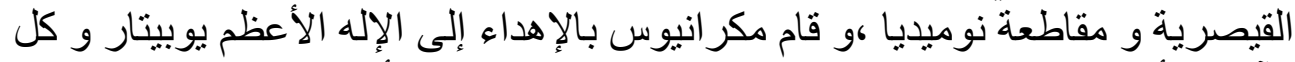

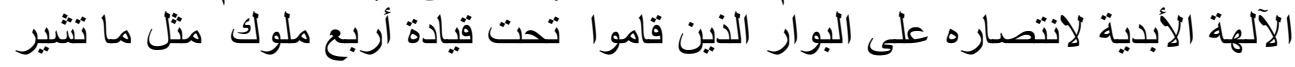

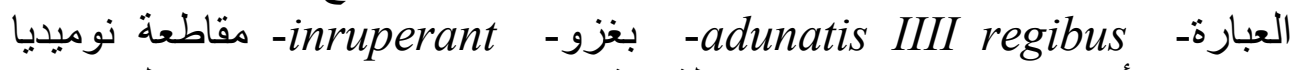

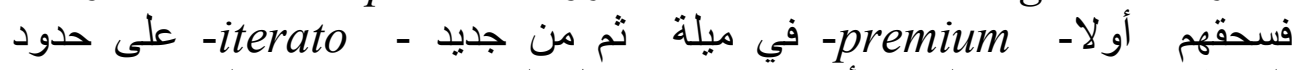

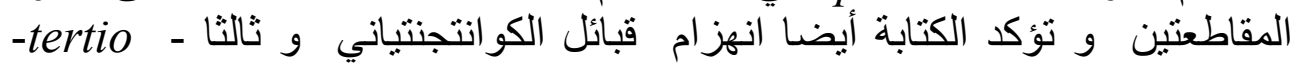

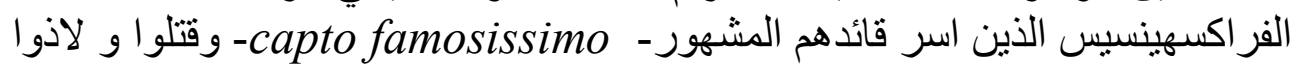
- caesis fugatisque - بالفر ار الفرين

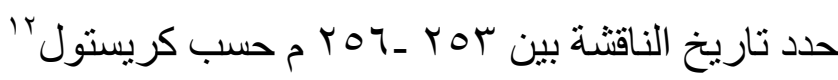

$$
\begin{aligned}
& \text { 4- ناقثنة سور الغز لان(وسط الجزائر) - Auzia }
\end{aligned}
$$

$\mathrm{Q}$ (uinto)G]argilioQ(uinti)f(ilio)eq(uiti)R(omano)/[pr]aef(ecto)co $\mathrm{h}$ (ortis)Asturum

$\operatorname{pr}($ ovinciae)Brit(t)a/[n](n)iaetrib(uno)co(hortis)Hisp(anorum)pr(o vinciae)Maur(etaniae)Cae(sariensis)/[a]mil(itis)praep(osito)coh(o rtis)sing(ularium)et

vex(illationis)/[e]qq(uitum)Mauror(um)interritorio/[A]uziensisipr aetendentium/dec(urioni)duarum coll(oniarum)Auzien/sis et Rusguniensis et pat(rono)/prov(inciae)ob insignem in ci/ves amorem etsingula/rem erga patriam adfec/tionem et quod eius vir/tute ac vigilantia $\mathrm{Fa} /$ raxen rebellis cum sa/tellibus suis fuerit/captus et interfectus/ordo col(oniae)Auziensis insidiis Bavarum $\mathrm{de} /$ ceptop(ecunia)f(ecit)d(e)d(icatum)vIIIkal(endas)/[A]priles) $\operatorname{pr}($ ovinciae)ccxxI

\footnotetext{
${ }^{12}$ Christol.M, « Prosopographie de Numidie », Ant.Af, 1976, p, 76 ${ }^{13}$ CIL,tome, VIII ,n 09047
} 


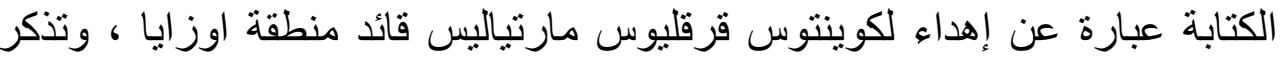

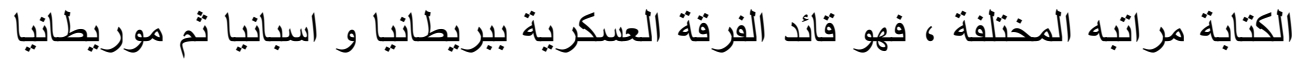

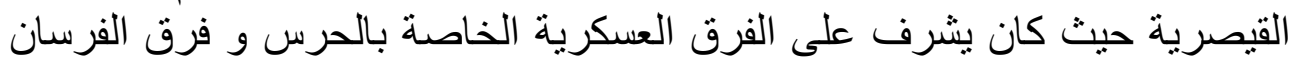

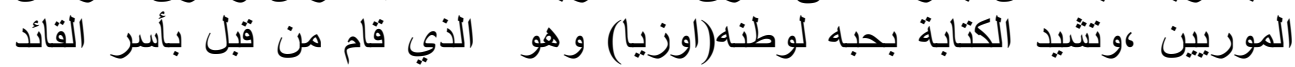

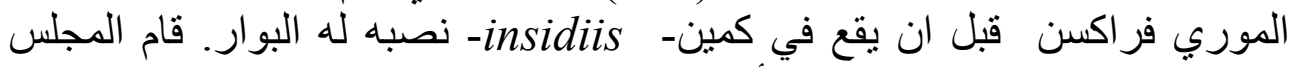

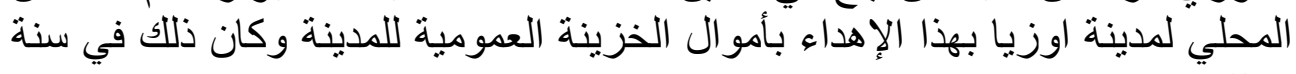
.

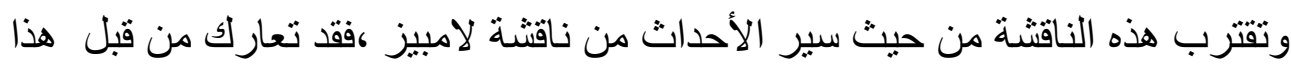

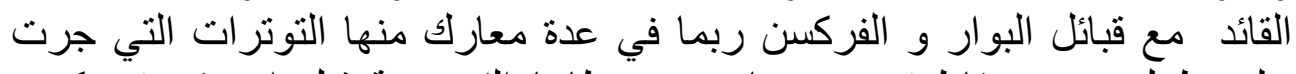

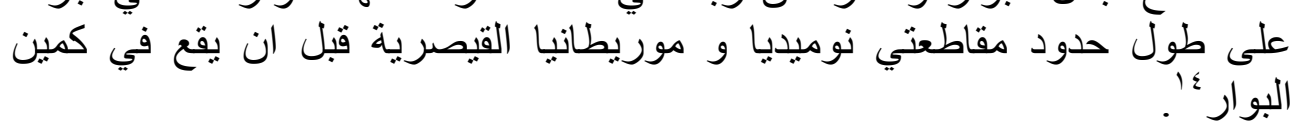

ه ناقتشة عين بوديب (نواحي سور الغزلان وسط الجزائر 10

I(ovi )O(ptimo) M(aximo)Gennisque diis[i]mmortalibus [vict]oriisque $\mathrm{d}$ (ominorum)n(ostrorum) invic[t]orum $\mathrm{M}$ (arcus)

Aur vitalis v(ir) e(gregius) $\mathrm{p}$ (raeses) $\mathrm{p}$ (rovinciae)

Maur(etaniae)Caesarien(sis)Ulp(ius)Castus dec(urio) alae Thracum ob Barbaros cesos et fusos v(otum)

$\mathrm{s}$ (olvit)(libens)a(nimo)[...]idus A ugusta a (nno) p(rovinciae)cc et $\mathrm{XV}$

تثتير الكتابة إلى احتفال حاكم موريطانيا القيصرية ماركوس اورليوس فتاليس و قائد

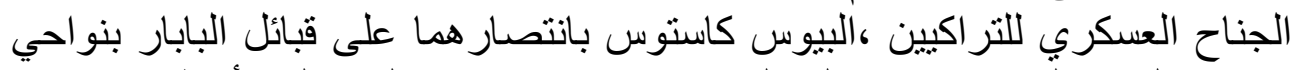

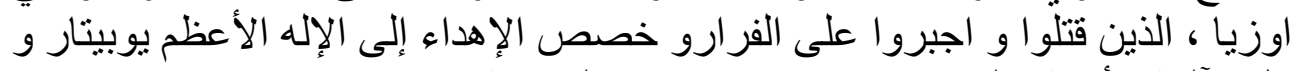

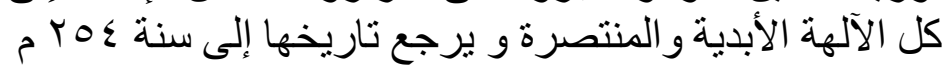

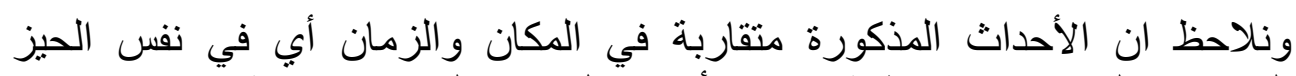

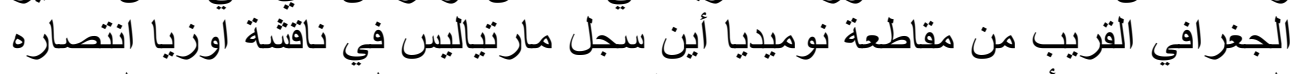

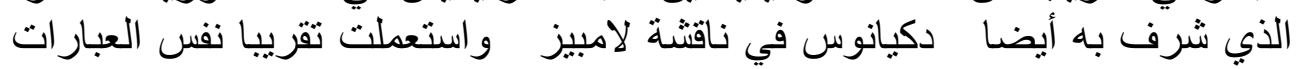
الدالة على سحق البوار و هي:

\footnotetext{
${ }^{14}$ Christol.M, op.cit p, 72

${ }^{15}$ CIL,tome, VIII ,n 20827
} 
- item gentilibus fraxinensibus...caesis fugatisque

$$
7 \text {-ناقثة المهدية (نو احي سطيف شرق الجزائر } 17
$$

..se si..../...Bavaribus.../...rebellibus et in/[p]riori

Praesidatu/[e]t post indicatu/(M)Cornel Octavianus/pr(aefectus).

Class(is) prae[t] (oriae).Misen(sis).fe]cit agens (gratias...

يتحدث نص الناقثنة عن إهداء قام به حاكم موريطانيا القيصرية ماركوس كرنليوس

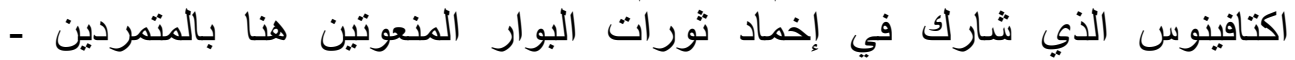

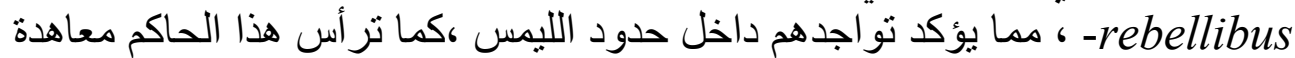

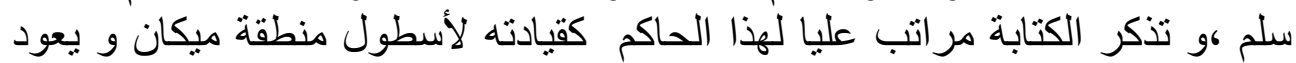

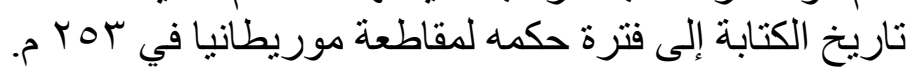

و قد وجد اسم هذا الحاكم في عدة نقوش، و تعطينا ناقثنة اكتشفت بشر شال تفاصيل

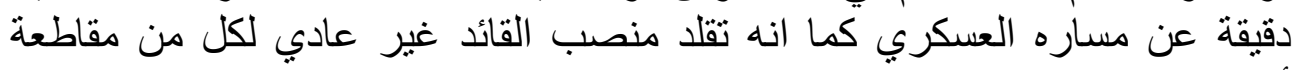
أفريكا و نوميديا و موريطانيا - mauretaniamque

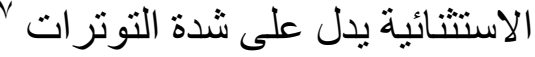

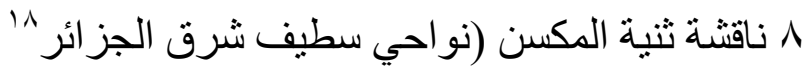

....Bavarum gentes quorum omnis mul

Titu[do prostrata est,interfectis Taganin Masmule et Fahem reg

Bus adpraehensis etiam afram/fa...vasamen et...inim con

Lectis rega[libus vota diis immortalibus Getulor [persolv]it $\operatorname{sul}[\mathrm{pi}]$

Eius sac...us

\footnotetext{
${ }^{16}$ ILS, Berolini ,1892-1916 ,n, 9006

${ }^{17}$ Pavis-Descurac Doisy.H , « M.Cornelius Octavianus et les révoltes indigènes du 3 siecle »,libyca,n1,1953pp180-187

${ }^{18}$ 18-ILS, n, 8959
} 


\section{1}

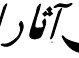

وتقترب هذه الناقشة أيضا من سابقتها في الزمان والمكان وتذكر أسماء ثلاثة ملوك

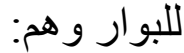

و تثير أيضا إلى العدد الكبير للبوار كما تبينه

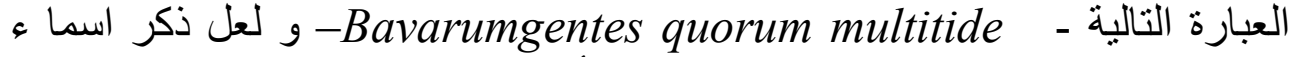
قادة البوار و إعطاءة هم صفة الملوك لدليل على أهمية هذه الثورات.

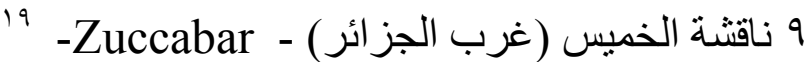

Diis( !)Patriis et Mauris/Conservatoribus/Aelius Aelianus v(ir) $\mathrm{p}$ (erfectissimus)/praeses provinciae/Mauretaniae caes(ariensis)/ob prostratam gentem/Bavarum Mesegneitsium/praedasque omnes ac fami/lias eorum abductas/votum solvit

تخص الكتابة إهداء قام به حاكم موريطانيا القيصرية ايليوس ايليانوس إلى الآلهة

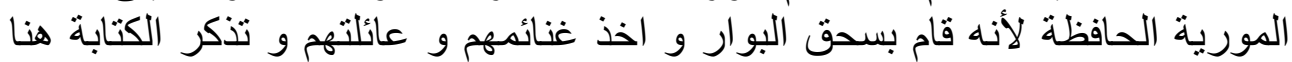

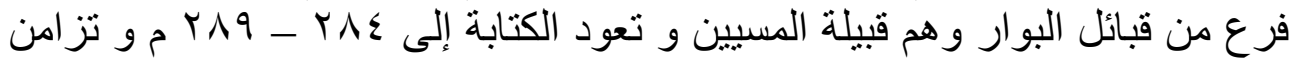

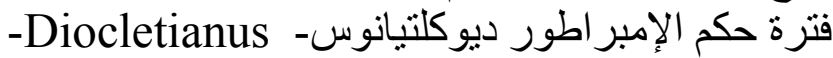

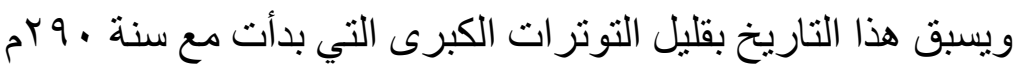

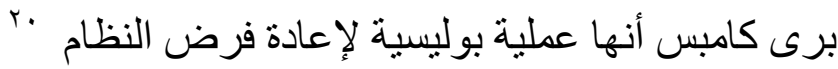
Zucchabar (غرب الجزائر) - ناقنتة مليانة

Di(i)s P[at]riis de /abusque Fortun(a)e/Reduci pro salute/a[t]que incolumitate/d(omini) $\mathrm{n}$ (ostri) $\mathrm{im}[\mathrm{p}$ (eratoris) $] \mathrm{C}[\mathrm{a}] \mathrm{e}$ (saris)/P(ublii)Licini G[a]llie[n]i/PiiFel(icis)Aug(usti)/M(arcus)Aurel(ius) Victor/v(ir)e(gregius)pr(a)eses prov(inciae)ccxxIIII/k(alendis)I( !)

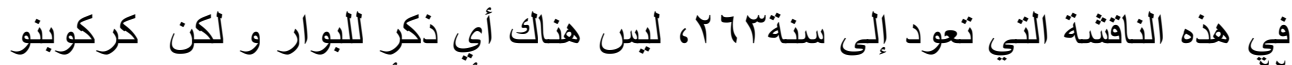

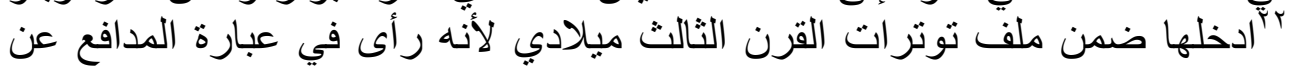

\footnotetext{
${ }^{19}$ CIL,tome,VIII, n, 21486

${ }^{20}$ Camps.G, « Les Bavares, » encyclopédie Berbère, 1991, p1395

${ }^{21}$ AE, n, 1920, 00108

${ }^{22}$ Carcopino.J , « L’insurrection de 253, d'après une inscription de Miliana, » R.Af, 1919, pp369-383

CIL,tome,VIII, n, 09324
} 


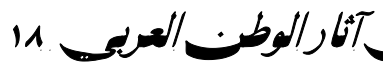

- دورا عسكريا لهذا الحاكم ماركوس اورليوس فكتور في -protector eiusالمقاطعة

ويخالف كريستول هذا الطرح ور أى في العبارة نوع من الوفاء الذي كان يوليه الحاكم

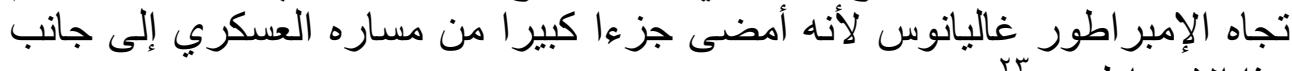
هذا الإمبر اطور.

وقد ننحاز أكثر إلى رأي كركوبنو ،ولكننا لا نستطيع تأكيد ذلك في غياب أي إثارة

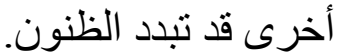

ناقثنة شرشال- (وسط الجز ائر) Caesarea-C

Iovi Optimo Maximo/ceterisque dis/immortalibus/gratum referens/quod erasis funditus/babaris()Trantagnen/sibus secunda praeda/facta salvus et incolumis/cum omnib(us)militibus/dd(ominorum)nn(ostrorum)Diocletiani et Maximiani Augg(ustorum)/regressus/Aurel(ius)Ltua v(ir) $\mathrm{p}$ (erfectissimus) $\mathrm{p}$ (raeses) $\mathrm{p}$ (rovinciae) $\mathrm{M}$ (auretaniae)C(aesariensis)/votum libens posui

تتحدث الناقتة عن اهدءا قام به حاكم موريطانيا القيصرية اورليوس لتويا في عهد

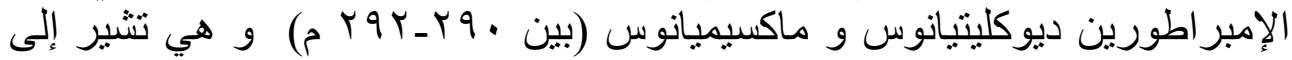

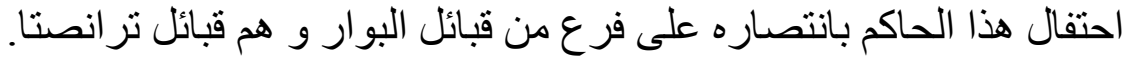

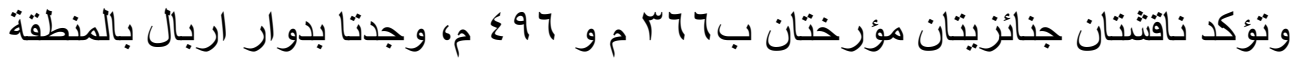

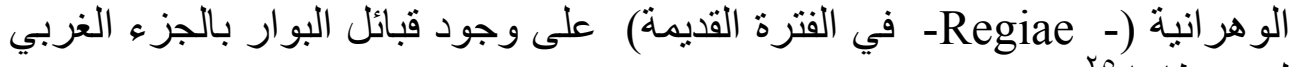
لموريطانيا

و هي تثير إلى ان هذين الثخصين كانا ضحية البوار كما تدل عليه العبارة الواردة في

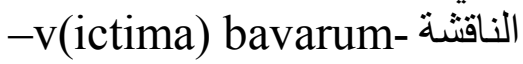

$1 \mathrm{D}$ (is) $\mathrm{M}$ (anibus) $\mathrm{S}$ (acrum)/M(arco)Lollio $\mathrm{Sa} /$ bino marito/amantissim/o qui a vi Bav/arus passus/ est vixit an(nos)/xxxI Aurelia/Mai(i)orica ti/tul(m) fecit

\footnotetext{
${ }^{23}$ Christol.M et Salama.P «,Une nouvelle inscription d'Aioun Sbiba concernant l'insurrection Mauretanienne, dite de 253 »,Cahiers du Centre Gustave Glotz,v 12,2001,pp253-267

${ }^{24}$ CIL,tome,VIII, n, 09324

${ }^{25}$ CILVIII ,21644 et 21630
} 
an(no)p(rovinciae)ccc/[xx]vII( ?)Ma(nlio ?)Lollio F/[3]so

$\operatorname{vix(it)an(nos)XX~[1]~}$

$2 \mathrm{D}$ (is) $\mathrm{M}$ (anibus)S(acrum)/P(ublio)(A)elio

Felici/amantissimo/[qui]vi

Bavarum/perfect[u]s( !)[es] $<\mathrm{t}=>/$ vixitan(nis)/xxxma[er3]/[3]aram [atq(ue)]t/itulu(m)fece[runt]/pro(vinciae)cc

$$
\text { عيون سبيبة (المنطقة الو هر انية غرب الجزائر بrان }
$$

$\mathrm{D}$ (is) S(alutaribus) M(auris) / [M(arcus)] Aur(elius) Victor v(ir) e(gregius) / proc(urator) praeses prov (inciae) / Maur(etaniae) Caes[ariens(is)] / [fo]nte[m 3]/cial[3] / ceria[3] / [1]nius [

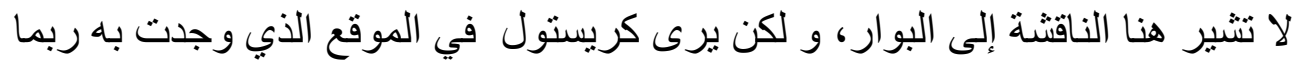

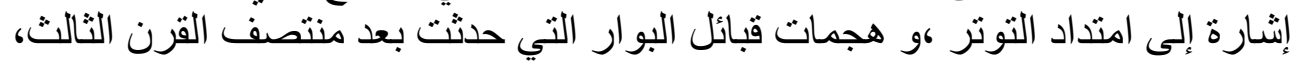

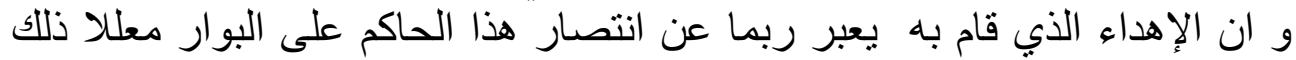

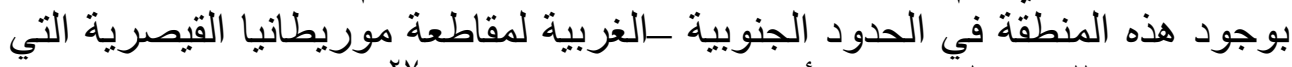

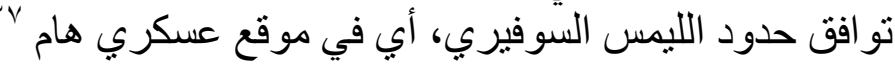

و على ضوء المصادر و النقوش يمكننا تأكيد وجود توتر ات قام بها قبائل البوار خاصة في مقاطعة موريطانيا القيصرية.

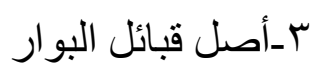

يمكننا ان نستخلص من خلال الأبحاث التي أقيمت حول قبائل البو ار وجود اقتر احين - هناك من يرى أمثال اوزينا (Euzennat)وكورتو ا- ( Courtois)

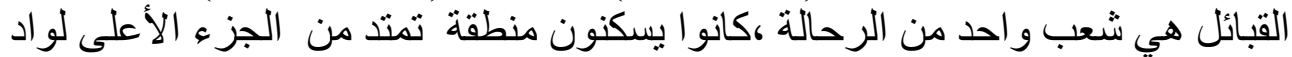

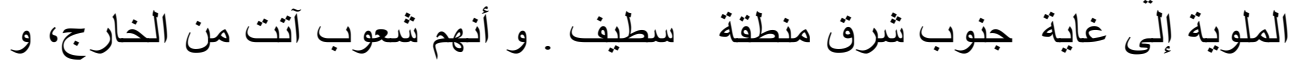

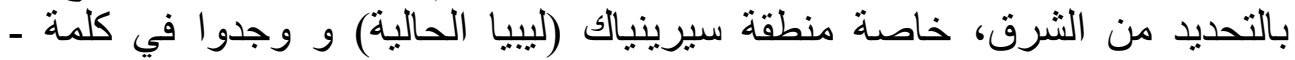

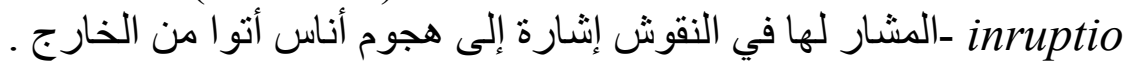

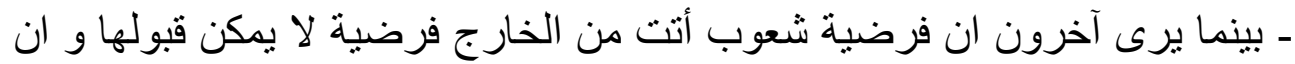
كلمة - inruptio -تشير إلى تحرك شعوب داخل المقاطعة ،خرجت من مجالها

\footnotetext{
${ }^{26}$ AE 2001, 02137

${ }^{27}$ Christol.M et Salama.P,Une nouvelle inscription d'Aioun Sbiba , op cit, ,pp253-267

${ }^{28}$ Euzzenat.M, « Les troubles de Mauretanie, », C.R.A.I.B.L, 1984, pp372-

393 ;Courtois.Ch,Les vandales et l'Afrique romaine,Paris, 1955
} 


\section{دراسات فحـ آثار/الوط. العربحس 11}

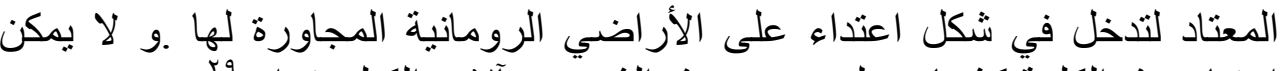

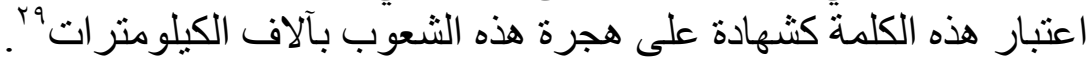

ولا تشير المصادر و النقوش إلى أنهم شعب مهاجر وهو ما ير اه معظم الباحثين. ويقسمهم كامبس إلى مجمو عتين عرقيتين مختلفتين، مجمو عة شرقية كانت تقطن

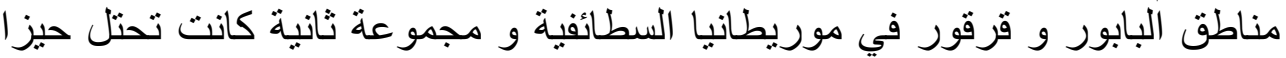

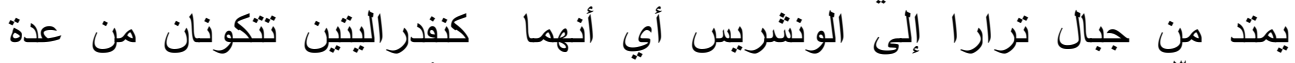

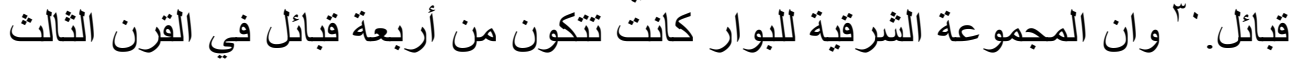

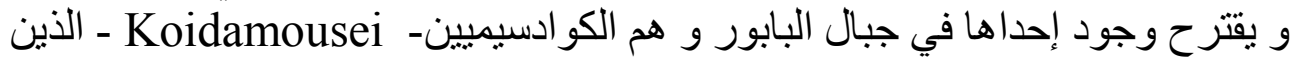

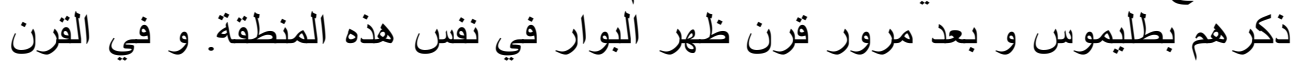
الخامس نجد قبائل اوكاتمي- Ucatami - الذين سيصبحون في في فئرن الفترة الإسلامية

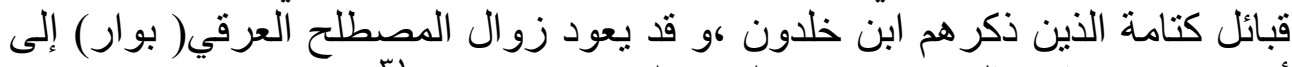

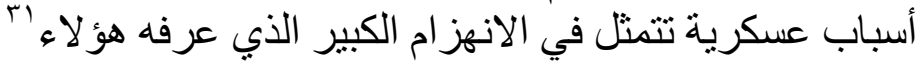

البذهب كامبس بعيدا في تفسيره الاتمولوجي لكلمة بربر التي حسبه قد تعود إلى قبائل

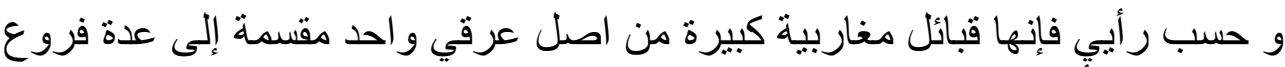

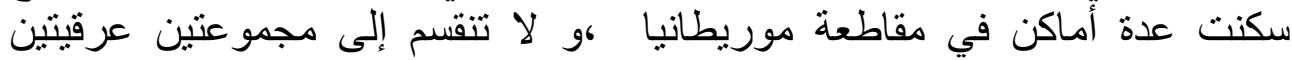

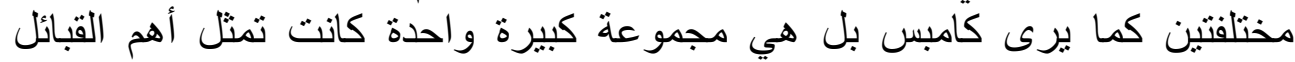

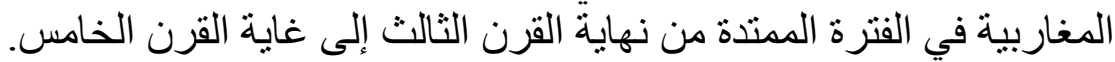

ع شورات البوار(الحوصلة)

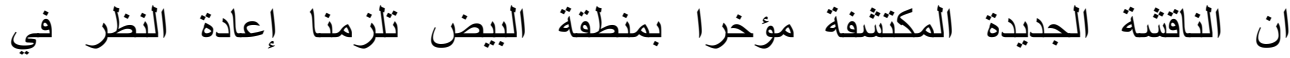

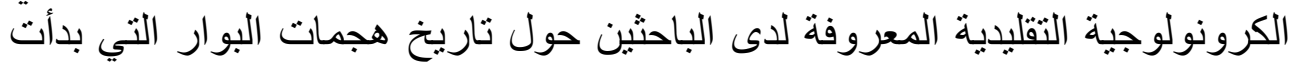

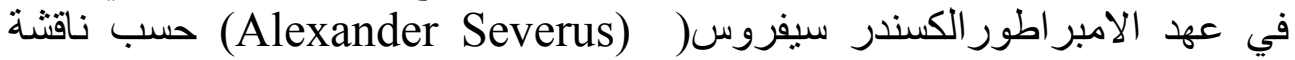
وليلي ،لان نص ناقتة البيض بعود بنا إلى فترة اسبق ، ترجع إلى الإمبراطور

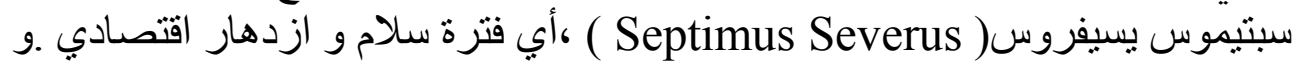

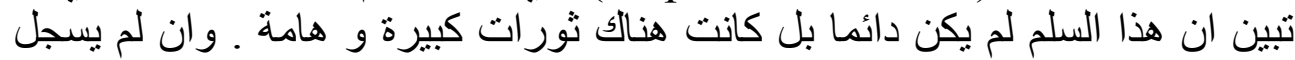

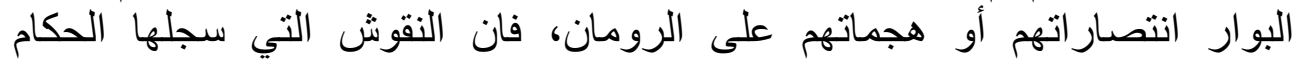

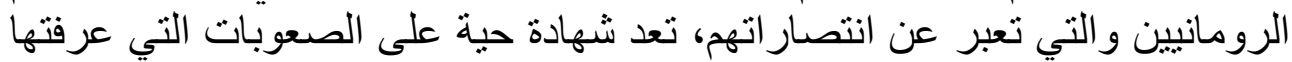

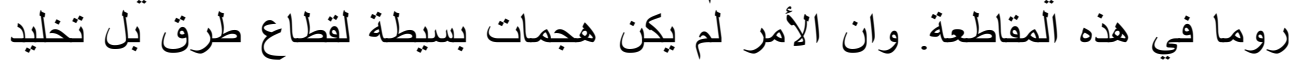

\footnotetext{
${ }^{29}$ Kotula.T, «Les Africains et la domination de Rome, », Dialogues d'histoire ancienne, vol, 2, 1976, p, 351

${ }^{30}$ Camps.G,op.cit,pp 1394-1399

${ }^{31}$ Camps.G ,ibid, p, 1397

${ }^{32}$ Camps.G,ibid,p1398
} 


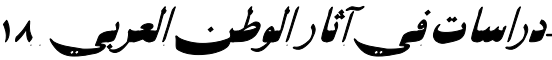

الحاكم لانتصاره دليل على انه كان في مواجهة قوة منظمة عسكريا استوجبت قدوم الحاكم لإخمادها الذي حرص لإن على إعلان ذللك في نص الكتابة.

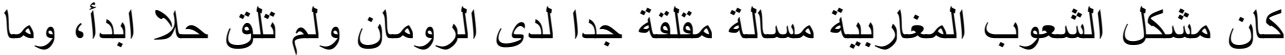

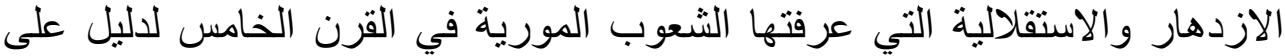

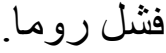

فهذه الثورات هي نتيجة التوسع الروماني الذي نتج عنه تصدي محلي لم بستطع

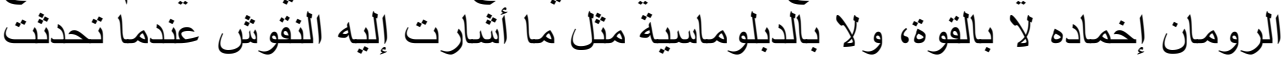

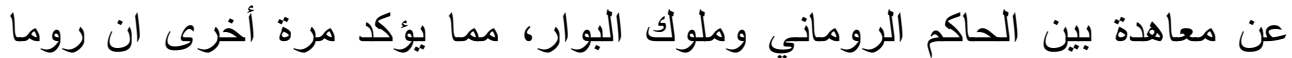

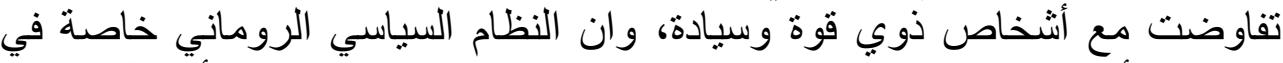

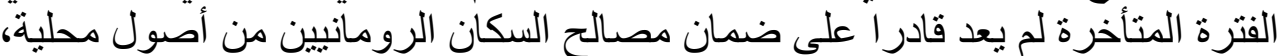

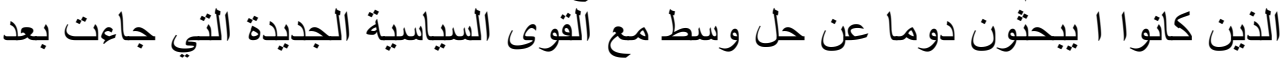

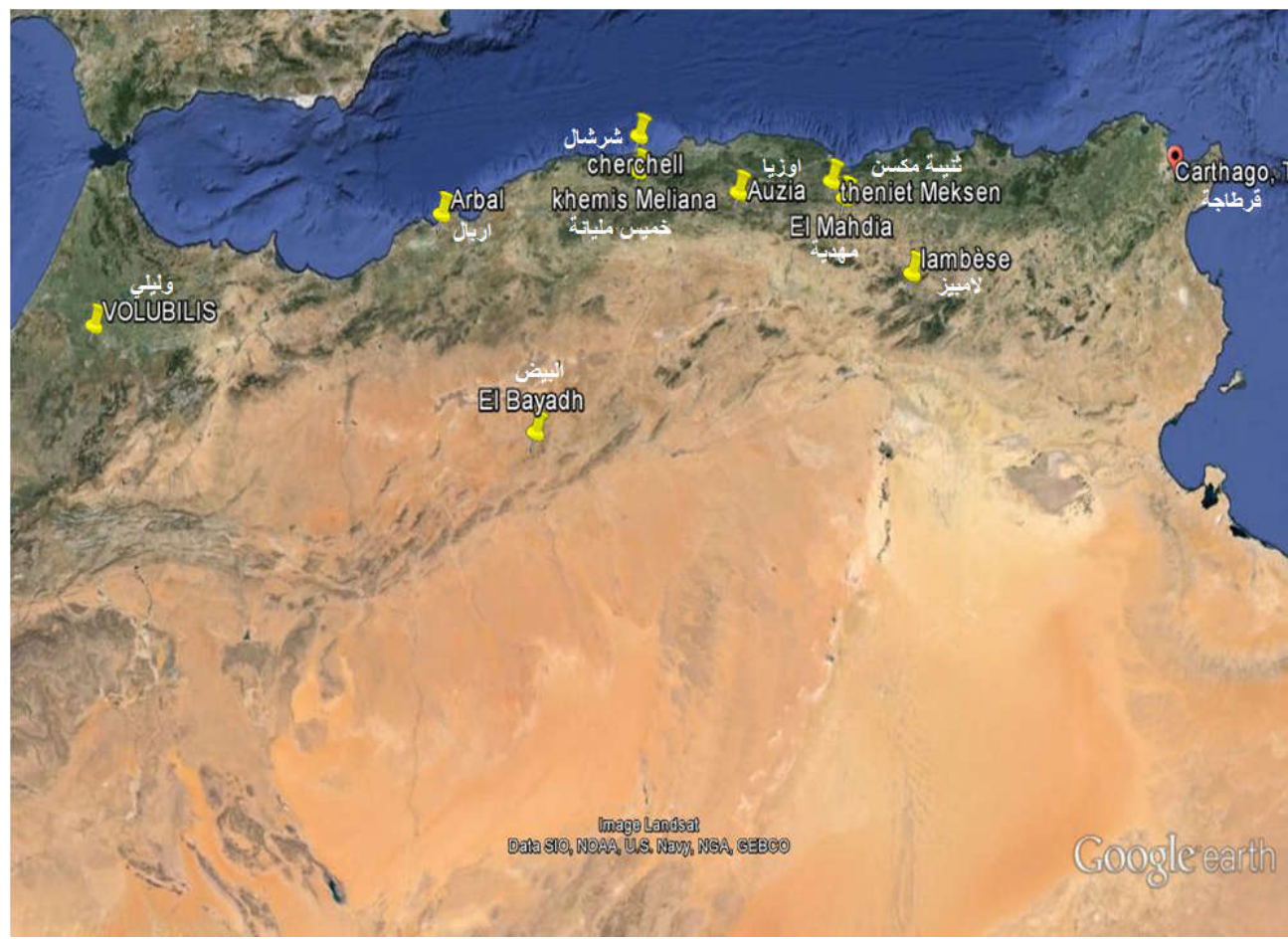

المناطق التي عرفت تو اجد قبائل البوار (حب النقوش) 
-Année Epigraphique

- Ant.Af, Antiquités Africaines

-CIL, Corpus Inscriptionum Latinarum,

- C.R.A.I.B.L, Comptes rendus de l'Académie des Inscriptions et des Belles Lettres

- IAM, Inscriptions Antiques du Maroc

- ILS, Inscriptiones Latinae Selectae

-R.Af, Revue Africaine

$$
\begin{aligned}
& \text { * قانون كتابة النص اللاتيني } \\
& \text { ا } \\
& \text { r ( ) ( ) مختصر بستحيل كتابته }
\end{aligned}
$$

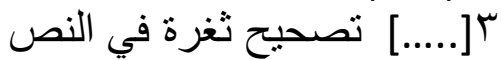

$$
\begin{aligned}
& \text { ع [.......][] طرق في الحجر المنقوش فئه } \\
& \text { ד } \\
& \text { T } \\
& \text { 人 > > V }
\end{aligned}
$$

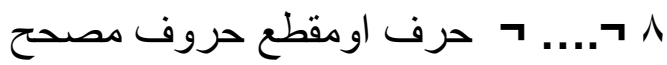

$$
\begin{aligned}
& \text { A حرف محرف واضح } \\
& \text {. } \\
& \text { 11 }
\end{aligned}
$$

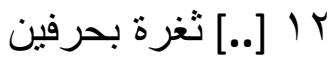

$$
\begin{aligned}
& \text { ع آ [----] تغرة بطول غير محدد على نفس السطر } \\
& \text { ؟ ([---|---] ثغرة بطول غير محدد على سطرين }
\end{aligned}
$$

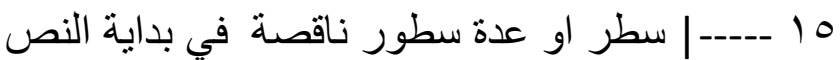

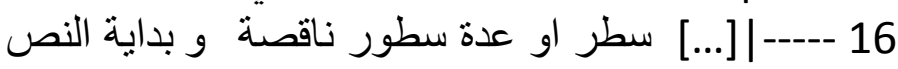

$$
\begin{aligned}
& \text { 17|----- نقص سطر او عدة سطور في اخر النص }
\end{aligned}
$$

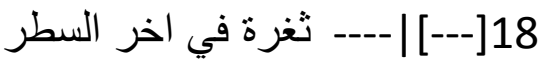

$$
\begin{aligned}
& 19 \text { * حروف ومقاطع نقتت مرنين بالخطأ }
\end{aligned}
$$




\title{
Bavares tribes in ancient Maghreb through sources and Latin inscription
}

\section{Mehentel Djahida}

\begin{abstract}
:
The Bavares are one of the biggest Maghreb tribes, known in the ancient period; particularly in the province of Caesarea Mauritania.the ancient sources have reminded them in a later period, around the fourth century AD. Plinus, roman writter did not remind them in his talk about the natural history of Africa .By the Latin inscriptions discovered in several areas, mostly in Algeria. We can reconsider been said before about these tribes, especially after the discovery in El-Bayed, area in 2014 (south western Algeria) a new inscription which give us a new chronological order. By sources and inscriptions, we can confirm the big presence of these tribes and their great resistance to the Roman occupation.
\end{abstract}

Key words:

Tribes, Bavares, ancient, Maghreb, Caesarea Mauretania, late period, sources, inscriptions, ElBayed, resistance, roman

\footnotetext{
- Degree/ professor Institution/ Archeology institute, Algiers University II djahidameh@gmail.com
} 